\title{
INTEGRAL MEANS OF CERTAIN MULTIVALENT FUNCTIONS
}

\author{
S. SÜMER EKER, H. ÖZLEM GÜNEY, AND SHIGEYOSHI OWA
}

Received 9 December 2005; Accepted 2 May 2006

For analytic and multivalent functions $f(z)$ and $p(z)$ in the open unit disk $\mathbb{U}$, a subordination theorem due to Littlewood (1925) which was called the integral mean is applied. Some simple examples for our results are also considered.

Copyright $\odot 2006$ Hindawi Publishing Corporation. All rights reserved.

\section{Introduction}

Let $\mathscr{A}_{p, n}$ denote the class of functions $f(z)$ of the form

$$
f(z)=z^{p}+\sum_{k=p+n}^{\infty} a_{k} z^{k} \quad(p, n \in \mathbb{N}:=\{1,2,3, \ldots\})
$$

which are analytic and multivalent in the open unit disk $\mathbb{U}=\{z \in \mathbb{C}:|z|<1\}$. A function $f(z)$ belonging to $\mathscr{A}_{p, n}$ is called multivalently starlike of order $\alpha$ in $\mathbb{U}$ if it satisfies the inequality

$$
\operatorname{Re}\left(\frac{z f^{\prime}(z)}{f(z)}\right)>\alpha \quad(z \in \mathbb{U})
$$

for some $\alpha(0 \leq \alpha<p)$. Also, a function $f(z) \in \mathscr{A}_{p, n}$ is said to be multivalently convex of order $\alpha$ in $\mathbb{U}$ if it satisfies the inequality

$$
\operatorname{Re}\left(1+\frac{z f^{\prime \prime}(z)}{f^{\prime}(z)}\right)>\alpha \quad(z \in \mathbb{U})
$$

for some $\alpha(0 \leq \alpha<p)$. We denote by $\mathscr{S}_{p, n}^{*}(\alpha)$ and $\mathscr{K}_{p, n}(\alpha)$ the class of functions $f(z) \in$ $A_{p, n}$ which are multivalently starlike of order $\alpha$ and multivalently convex of order $\alpha$, respectively. We note that

$$
f \in \mathscr{K}_{p, n}(\alpha) \Longleftrightarrow \frac{z f^{\prime}}{p} \in \mathscr{Y}_{p, n}^{*}(\alpha) .
$$

Hindawi Publishing Corporation

International Journal of Mathematics and Mathematical Sciences

Volume 2006, Article ID 90921, Pages 1-9

DOI 10.1155/IJMMS/2006/90921 
2 Integral means of certain multivalent functions

For functions $f(z)$ belonging to the classes $\mathscr{S}_{p, n}^{*}(\alpha)$ and $\mathscr{K}_{p, n}(\alpha)$, Owa [4] has shown the following coefficient inequalities.

Theorem 1.1. If a function $f(z) \in \mathscr{A}_{p, n}$ satisfies

$$
\sum_{k=p+n}^{\infty}(k-\alpha)\left|a_{k}\right| \leq p-\alpha
$$

for some $\alpha(0 \leq \alpha<p)$, then $f(z) \in \mathscr{Y}_{p, n}^{*}(\alpha)$.

Theorem 1.2. If a function $f(z) \in \mathscr{A}_{p, n}$ satisfies

$$
\sum_{k=p+n}^{\infty} k(k-\alpha)\left|a_{k}\right| \leq p(p-\alpha)
$$

for some $\alpha(0 \leq \alpha<p)$, then $f(z) \in \mathcal{K}_{p, n}(\alpha)$.

For analytic functions $f(z)$ and $g(z)$ in $\mathbb{U}, f(z)$ is said to be subordinate to $g(z)$ in $\mathbb{U}$ if there exists an analytic function $w(z)$ in $\mathbb{U}$ with $w(0)=0$ and $|w(z)|<1(z \in \mathbb{U})$ such that $f(z)=g(w(z))$. We denote this subordination by

$$
f(z) \prec g(z) \quad(\text { cf. Duren [1] }) .
$$

The following subordination theorem will be required in our present investigation.

Theorem 1.3 (Littlewood [3]). If $f(z)$ and $g(z)$ are analytic in $\mathbb{U}$ with $f(z) \prec g(z)$, then for $\mu>0$ and $z=r e^{i \theta}(0<r<1)$,

$$
\int_{0}^{2 \pi}|f(z)|^{\mu} d \theta \leqq \int_{0}^{2 \pi}|g(z)|^{\mu} d \theta
$$

Applying Theorem 1.3 by Littlewood [3], Sekine et al. [5] have considered some integral means inequalities for certain analytic functions. Furthermore, Güney et al. [2] have studied integral means inequalities for multivalent functions.

In the present paper, we discuss the integral means inequalities for multivalent functions which are the generalization of the paper by Güney et al. [2].

\section{Integral means inequalities for $f(z)$ and $p(z)$}

In this section, we discuss the integral means inequalities for $f(z) \in \mathscr{A}_{p, n}$ and $p(z)$ defined by

$$
p(z)=z^{p}+\sum_{s=1}^{m} b_{s j-(s-1) p} z^{s j-(s-1) p} \quad(j \geqq n+p, n \in \mathbb{N}, m \geq 2) .
$$

We begin by giving the following lemma due to Sekine et al. [5].

Lemma 2.1. Let $P_{m}(t)$ denote the polynomial of degree $m$ of the form

$$
P_{m}(t)=c_{1} t^{m}-c_{2} t^{m-1}-\cdots-c_{m-1} t^{2}-c_{m} t-d \quad(t \geqq 0),
$$


S. Sümer Eker et al. 3

where $c_{i}(i=1,2, \ldots, m)$ are arbitrary positive constants and $d \geqq 0$. Then $P_{m}(t)=0$ has unique solution for $t>0$. If the solution is given by $t_{0}, P_{m}(t)<0$ for $0<t<t_{0}$ and $P_{m}(t)>0$ for $t>t_{0}$.

Our first result for integral means is contained in the following.

Theorem 2.2. Let $f(z) \in \mathscr{A}_{p, n}$ and let $p(z)$ be given by (2.1). If $f(z)$ satisfies

$$
\sum_{k=p+n}^{\infty}\left|a_{k}\right| \leqq\left|b_{m j-(m-1) p}\right|-\sum_{s=1}^{m-1}\left|b_{s j-(s-1) p}\right|
$$

with

$$
\sum_{s=1}^{m-1}\left|b_{s j-(s-1) p}\right|<\left|b_{m j-(m-1) p}\right|,
$$

and there exists an analytic function $w(z)$ such that

$$
\sum_{s=1}^{m} b_{s j-(s-1) p} w(z)^{s(j-p)}-\sum_{k=p+n}^{\infty} a_{k} z^{k-p}=0,
$$

then, for $\mu>0$ and $z=r e^{i \theta}(0<r<1)$,

$$
\int_{0}^{2 \pi}|f(z)|^{\mu} d \theta \leqq \int_{0}^{2 \pi}|p(z)|^{\mu} d \theta
$$

Proof. Putting $z=r e^{i \theta}(0<r<1)$, it follows that

$$
\begin{aligned}
& \int_{0}^{2 \pi}|f(z)|^{\mu} d \theta=r^{p \mu} \int_{0}^{2 \pi}\left|1+\sum_{k=p+n}^{\infty} a_{k} z^{k-p}\right|^{\mu} d \theta, \\
& \int_{0}^{2 \pi}|p(z)|^{\mu} d \theta=r^{p \mu} \int_{0}^{2 \pi}\left|1+\sum_{s=1}^{m} b_{s j-(s-1) p} z^{s j-s p}\right|^{\mu} d \theta .
\end{aligned}
$$

Applying Theorem 1.3, we have to show that

$$
1+\sum_{k=p+n}^{\infty} a_{k} z^{k-p} \prec 1+\sum_{s=1}^{m} b_{s j-(s-1) p} z^{s(j-p)} .
$$

Let us define the function $w(z)$ by

$$
1+\sum_{s=1}^{m} b_{s j-(s-1) p}\{w(z)\}^{s(j-p)}=1+\sum_{k=p+n}^{\infty} a_{k} z^{k-p}
$$


4 Integral means of certain multivalent functions

or by

$$
\sum_{s=1}^{m} b_{s j-(s-1) p}\{w(z)\}^{s(j-p)}=\{w(z)\}^{j-p}\left(\sum_{s=1}^{m} b_{s j-(s-1) p}\{w(z)\}^{(s-1)(j-p)}\right)=\sum_{k=p+n}^{\infty} a_{k} z^{k-p} .
$$

Thus, it follows that

$$
\{w(0)\}^{j-p}\left(\sum_{s=1}^{m} b_{s j-(s-1) p}\{w(z)\}^{(s-1)(j-p)}\right)=0 .
$$

Therefore, if there exists an analytic function $w(z)$ which satisfies the equality (2.5), we can consider an analytic function $w(z)$ in $\mathbb{U}$ such that $w(0)=0$.

Further, we need to prove that this analytic function $w(z)$ satisfies $|w(z)|<1(z \in \mathbb{U})$ for

$$
\sum_{k=p+n}^{\infty}\left|a_{k}\right| \leqq\left|b_{m j-(m-1) p}\right|-\sum_{s=1}^{m-1}\left|b_{s j-(s-1) p}\right| \quad\left(\sum_{s=1}^{m-1}\left|b_{s j-(s-1) p}\right|<\left|b_{m j-(m-1) p}\right|\right) .
$$

From the equality (2.5), we know that

$$
\begin{aligned}
& \mid b_{m j-(m-1) p} w(z)^{m(j-p)}+b_{(m-1) j-(m-2) p} w(z)^{(m-1)(j-p)}+b_{(m-2) j-(m-3) p} w(z)^{(m-2)(j-p)} \\
& +\cdots+b_{2 j-p} w(z)^{2(j-p)}+b_{j} w(z)^{j-p}\left|\leqq \sum_{k=p+n}^{\infty}\right| a_{k} z^{k-p}\left|<\sum_{k=p+n}^{\infty}\right| a_{k} \mid
\end{aligned}
$$

for $z \in \mathbb{U}$, so that

$$
\left|b_{m j-(m-1) p}\right|\left|w(z)^{m(j-p)}\right|-\sum_{s=1}^{m-1}\left|b_{s j-(s-1) p}\right||w(z)|^{s(j-p)}-\sum_{k=p+n}^{\infty}\left|a_{k}\right|<0
$$

for $z \in \mathbb{U}$.

Putting $t=|w(z)|^{j-p}(t \geqq 0)$, we define the polynomial $P(t)$ of degree $m$ by

$$
P(t)=\left|b_{m j-(m-1) p}\right| t^{m}-\sum_{s=1}^{m-1}\left|b_{s j-(s-1) p}\right| t^{s}-\sum_{k=p+n}^{\infty}\left|a_{k}\right|
$$

By means of Lemma 2.1, if $P(1) \geqq 0$, we have $t<1$ for $P(t)<0$. Hence for $|w(z)|<1(z \in$ $\mathbb{U})$, we need the following inequality:

$$
P(1)=\left|b_{m j-(m-1) p}\right|-\sum_{s=1}^{m-1}\left|b_{s j-(s-1) p}\right|-\sum_{k=p+n}^{\infty}\left|a_{k}\right| \geqq 0
$$


S. Sümer Eker et al. 5

so that

$$
\sum_{k=p+n}^{\infty}\left|a_{k}\right| \leqq\left|b_{m j-(m-1) p}\right|-\sum_{s=1}^{m-1}\left|b_{s j-(s-1) p}\right|
$$

Therefore, the subordination in (2.8) holds true, and this evidently completes the proof of Theorem 2.2.

Theorem 2.2 gives us the following corollary.

Corollary 2.3. Let $f(z) \in \mathscr{A}_{p, n}$ and let $p(z)$ be given by (2.1). If $f(z)$ satisfies the conditions of Theorem 2.2, then for $0<\mu \leqq 2$ and $z=r e^{i \theta}(0<r<1)$,

$$
\int_{0}^{2 \pi}|f(z)|^{\mu} d \theta \leqq 2 \pi r^{p \mu}\left(1+\sum_{s=1}^{m}\left|b_{s j-(s-1) p}\right|^{2} r^{2 s(j-p)}\right)^{\mu / 2}<2 \pi\left(1+\sum_{s=1}^{m}\left|b_{s j-(s-1) p}\right|^{2}\right)^{\mu / 2} .
$$

Furthermore, $f(z) \in \mathscr{H}^{q}(\mathbb{U})$ for $0<q \leqq 2$, where $\mathscr{H}^{q}$ denotes the Hardy space.

Proof. Since

$$
\int_{0}^{2 \pi}|p(z)|^{\mu} d \theta=\int_{0}^{2 \pi}\left|z^{p}\right|^{\mu}\left|1+\sum_{s=1}^{m} b_{s j-(s-1) p} z^{s(j-p)}\right|^{\mu} d \theta
$$

applying Hölder inequality for $0<\mu<2$, we obtain that

$$
\begin{aligned}
\int_{0}^{2 \pi}|p(z)|^{\mu} d \theta & \leqq\left(\int_{0}^{2 \pi}\left(|z|^{p \mu}\right)^{2 /(2-\mu)} d \theta\right)^{(2-\mu) / 2}\left\{\int_{0}^{2 \pi}\left(\left|1+\sum_{s=1}^{m} b_{s j-(s-1) p} z^{s(j-p)}\right|^{\mu}\right)^{2 / \mu} d \theta\right\}^{\mu / 2} \\
& =\left(r^{2 p \mu /(2-\mu)} \int_{0}^{2 \pi} d \theta\right)^{(2-\mu) / 2}\left(\int_{0}^{2 \pi}\left|1+\sum_{s=1}^{m} b_{s j-(s-1) p} z^{s(j-p)}\right|^{2} d \theta\right)^{\mu / 2} \\
& =\left(2 \pi r^{2 p \mu /(2-\mu)}\right)^{(2-\mu) / 2}\left\{2 \pi\left(1+\sum_{s=1}^{m}\left|b_{s j-(s-1) p}\right|^{2} r^{2 s(j-p)}\right)\right\}^{\mu / 2} \\
& =2 \pi r^{p \mu}\left(1+\sum_{s=1}^{m}\left|b_{s j-(s-1) p}\right|^{2} r^{2 s(j-p)}\right)^{\mu / 2}<2 \pi\left(1+\sum_{s=1}^{m}\left|b_{s j-(s-1) p}\right|^{2}\right)^{\mu / 2} .
\end{aligned}
$$

Further, it is easy to see that, for $\mu=2$,

$$
\int_{0}^{2 \pi}|f(z)|^{2} d \theta \leqq 2 \pi r^{2 p}\left(1+\sum_{s=1}^{m}\left|b_{s j-(s-1) p}\right|^{2} r^{2 s(j-p)}\right)<2 \pi\left(1+\sum_{s=1}^{m}\left|b_{s j-(s-1) p}\right|^{2}\right) .
$$


6 Integral means of certain multivalent functions

From the above, we also have that, for $0<\mu \leqq 2$,

$$
\sup _{z \in U} \frac{1}{2 \pi} \int_{0}^{2 \pi}|f(z)|^{\mu} d \theta \leqq\left(1+\sum_{s=1}^{m}\left|b_{s j-(s-1) p}\right|^{2}\right)<\infty
$$

which observes that $f(z) \in H^{2}(U)$. Noting that $H^{q} \subset H^{r}(0<r<q<\infty)$, we complete the proof of Corollary 2.3.

Example 2.4. Let $f(z) \in \mathscr{A}_{p, n}$ satisfy the coefficient inequality (1.5) and for $m=2$,

$$
p(z)=z^{p}+\frac{n}{p+n-\alpha} \varepsilon_{1} z^{j}+\varepsilon_{2} z^{2 j-p}
$$

and for $m \geq 3$,

$$
p(z)=z^{p}+\frac{n t^{m-2}}{p+n-\alpha} \varepsilon_{1} z^{j}+\sum_{s=2}^{m-1} \frac{n t^{(m-1)-s}(1-t)}{p+n-\alpha} \varepsilon_{s} z^{s j-(s-1) p}+\varepsilon_{m} z^{m j-(m-1) p},
$$

where $\left|\varepsilon_{i}\right|=1$ for all $i \in \mathbb{N}$ and $0 \leq \alpha<p$. Then, for $m=2$,

$$
b_{j}=\frac{n}{p+n-\alpha} \varepsilon_{1}, \quad b_{2 j-p}=\varepsilon_{2}
$$

and for $m \geq 3$,

$$
b_{j}=\frac{n t^{m-2}}{p+n-\alpha} \varepsilon_{1}, \quad b_{s j-(s-1) p}=\frac{n t^{(m-1)-s}(1-t)}{p+n-\alpha} \varepsilon_{s} \quad\{s=2,3, \ldots, m-1\},
$$

and $b_{m j-(m-1) p}=\varepsilon_{m}$.

Güney et al. [2] have shown the above example for the case $m=2$. By virtue of (1.5), we observe that, for $m \geqq 3$,

$$
\begin{aligned}
\sum_{k=p+n}^{\infty}\left|a_{k}\right| & \leqq \frac{p-\alpha}{p+n-\alpha}=1-\sum_{s=2}^{m-1} \frac{n t^{(m-1)-s}(1-t)}{p+n-\alpha}-\frac{n t^{m-2}}{p+n-\alpha} \\
& =\left|b_{m j-(m-1) p}\right|-\sum_{s=2}^{m-1}\left|b_{s j-(s-1) p}\right|-\left|b_{j}\right| .
\end{aligned}
$$


Therefore, $f(z)$ and $p(z)$ satisfy the conditions in Theorem 2.2. Thus, we have for $0<\mu \leqq$ 2 and $z=r e^{i \theta}(0<r<1)$,

$$
\begin{aligned}
\int_{0}^{2 \pi} \mid & \left.f(z)\right|^{\mu} d \theta \\
& \leqq 2 \pi r^{p \mu}\left\{1+\left(\frac{n t^{m-2}}{p+n-\alpha}\right)^{2} r^{2(j-p)}+\sum_{s=2}^{m-1}\left(\frac{n t^{(m-1)-s}(1-t)}{p+n-\alpha}\right)^{2} r^{2 s(j-p)}+r^{2 m(j-p)}\right\}^{\mu / 2} \\
& <2 \pi\left\{2+\left(\frac{n t^{m-2}}{p+n-\alpha}\right)^{2}+\sum_{s=2}^{m-1}\left(\frac{n t^{(m-1)-s}(1-t)}{p+n-\alpha}\right)^{2}\right\}^{\mu / 2} .
\end{aligned}
$$

\section{Integral means for $f^{\prime}(z)$ and $p^{\prime}(z)$}

Using the same techniques in Theorem 2.2, we obtain the following theorem.

Theorem 3.1. Let $f(z) \in \mathscr{A}_{p, n}$ and let $p(z)$ be given by (2.1). If $f(z)$ satisfies

$$
\sum_{k=p+n}^{\infty} k\left|a_{k}\right| \leqq(m j-(m-1) p)\left|b_{m j-(m-1) p}\right|-\sum_{s=1}^{m-1}(s j-(s-1) p)\left|b_{s j-(s-1) p}\right|
$$

with

$$
(m j-(m-1) p)\left|b_{m j-(m-1) p}\right|>\sum_{s=1}^{m-1}(s j-(s-1) p)\left|b_{s j-(s-1) p}\right|,
$$

and there exists an analytic function $w(z)$ such that

$$
\sum_{s=1}^{m}(s j-(s-1) p) b_{s j-(s-1) p}\{w(z)\}^{s(j-p)}-\sum_{k=p+n}^{\infty} k a_{k} z^{k-p}=0,
$$

then, for $\mu>0$ and $z=r e^{i \theta}(0<r<1)$,

$$
\int_{0}^{2 \pi}\left|f^{\prime}(z)\right|^{\mu} d \theta \leqq \int_{0}^{2 \pi}\left|p^{\prime}(z)\right|^{\mu} d \theta
$$

Further, with the help of Hölder inequality, we have the following.

Corollary 3.2. Let $f(z) \in \mathscr{A}_{p, n}$ and let $p(z)(m \geqq 2)$ be given by (2.1). If $f(z)$ satisfies the conditions in Theorem 3.1, then for $0<\mu \leqq 2$ and $z=r e^{i \theta}(0<r<1)$,

$$
\begin{aligned}
\int_{0}^{2 \pi}\left|f^{\prime}(z)\right|^{\mu} d \theta & \leqq 2 \pi r^{(p-1) \mu}\left(p^{2}+\sum_{s=1}^{m}(s j-(s-1) p)^{2}\left|b_{s j-(s-1) p}\right|^{2} r^{2 s(j-p)}\right)^{\mu / 2} \\
& <2 \pi\left(p^{2}+\sum_{s=1}^{m}(s j-(s-1) p)^{2}\left|b_{s j-(s-1) p}\right|^{2}\right)^{\mu / 2} .
\end{aligned}
$$


8 Integral means of certain multivalent functions

Example 3.3. Let $f(z) \in \mathscr{A}_{p, n}$ satisfy the coefficient inequality (1.6) and for $m=2$,

$$
p(z)=z^{p}+\frac{n}{j(p+n-\alpha)} \varepsilon_{1} z^{j}+\frac{1}{2 j-p} \varepsilon_{2} z^{2 j-p}
$$

and for $m \geq 3$,

$$
\begin{aligned}
p(z)= & z^{p}+\frac{n t^{m-2}}{j(p+n-\alpha)} \varepsilon_{1} z^{j} \\
& +\sum_{s=2}^{m-1} \frac{n t^{(m-1)-s}(1-t)}{[s j-(s-1) p](p+n-\alpha)} \varepsilon_{s} z^{s-(s-1) p}+\frac{\varepsilon_{m}}{m j-(m-1) p} z^{m j-(m-1) p,}
\end{aligned}
$$

where $\left|\varepsilon_{i}\right|=1$ for all $i \in \mathbb{N}$ and $0 \leq \alpha<p$. Then for $m=2$,

$$
b_{j}=\frac{n}{j(p+n-\alpha)} \varepsilon_{1}, \quad b_{2 j-p}=\frac{1}{2 j-p} \varepsilon_{2}
$$

and for $m \geq 3$,

$$
\begin{gathered}
b_{j}=\frac{n t^{m-2}}{j(p+n-\alpha)} \varepsilon_{1}, \quad b_{s j-(s-1) p}=\frac{n t^{(m-1)-s}(1-t)}{[s j-(s-1) p](p+n-\alpha)} \varepsilon_{s}, \\
b_{m j-(m-1) p}=\frac{\varepsilon_{m}}{m j-(m-1) p} .
\end{gathered}
$$

Güney et al. [2] have shown the above example for the case $m=2$. Since, for $m \geqq 3$,

$$
\begin{aligned}
\sum_{k=p+n}^{\infty} k\left|a_{k}\right| & \leqq \frac{p-\alpha}{p+n-\alpha}=1-\sum_{s=2}^{m-1} \frac{n t^{(m-1)-s}(1-t)}{p+n-\alpha}-\frac{n t^{m-2}}{p+n-\alpha} \\
& =(m j-(m-1) p)\left|b_{m j-(m-1) p}\right|-(s j-(s-1) p) \sum_{s=2}^{m-1}\left|b_{s j-(s-1) p}\right|-j\left|b_{j}\right|,
\end{aligned}
$$

$f(z)$ and $p(z)$ satisfy the conditions in Theorem 3.1. Thus, by Corollary 3.2, we have for $0<\mu \leqq 2$ and $z=r e^{i \theta}(0<r<1)$,

$$
\begin{aligned}
& \int_{0}^{2 \pi}\left|f^{\prime}(z)\right|^{\mu} d \theta \\
& \quad \leqq 2 \pi r^{(p-1) \mu}\left\{p^{2}+\left(\frac{n t^{m-2}}{p+n-\alpha}\right)^{2} r^{2(j-p)}+\sum_{s=2}^{m-1}\left(\frac{n t^{(m-1)-s}(1-t)}{p+n-\alpha}\right)^{2} r^{2 s(j-p)}+r^{2 m(j-p)}\right\}^{\mu / 2} \\
& \quad<2 \pi\left\{p^{2}+1+\left(\frac{n t^{m-2}}{p+n-\alpha}\right)^{2}+\sum_{s=2}^{m-1}\left(\frac{n t^{(m-1)-s}(1-t)}{p+n-\alpha}\right)^{2}\right\}^{\mu / 2} .
\end{aligned}
$$


Remark 3.4. In the above theorems, if we take that $p=1$, we obtain the results by Sekine et al. [5]. Furthermore, if we take that $m=2,3$ in the above theorems and examples, we obtain the results of Güney et al. [2]. Therefore, the results of our paper are a generalization of the results in [2].

\section{References}

[1] P. L. Duren, Univalent Functions, Fundamental Principles of Mathematical Sciences, vol. 259, Springer, New York, 1983.

[2] H. Ö. Güney, S. S. Eker, and S. Owa, Integral means of multivalent functions, Journal of Inequalities in Pure and Applied Mathematics 7 (2006), no. 1, 1-10, article 37.

[3] J. E. Littlewood, On inequalities in the theory of functions, Proceedings of the London Mathematical Society 23 (1925), 481-519.

[4] S. Owa, On certain classes of p-valent functions with negative coefficients, Simon Stevin 59 (1985), no. 4, 385-402.

[5] T. Sekine, S. Owa, and R. Yamakawa, Integral means of certain analytic functions, General Mathematics 13 (2005), no. 3, 99-108.

S. Sümer Eker: Department of Mathematics, Faculty of Science and Arts, University of Dicle, 21280 Diyarbakir, Turkey

E-mail address: sevtaps@dicle.edu.tr

H. Özlem Güney: Department of Mathematics, Faculty of Science and Arts, University of Dicle, 21280 Diyarbakir, Turkey

E-mail address: ozlemg@dicle.edu.tr

Shigeyoshi Owa: Department of Mathematics, Kinki University, Higashi-Osaka, Osaka 577-8502, Japan

E-mail addresses: shige21@ican.zaq.ne.jp; owa@math.kindai.ac.jp 


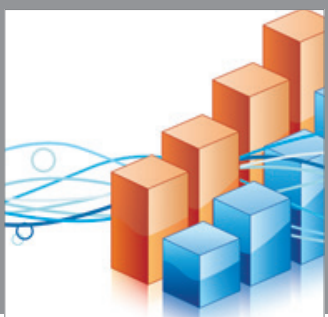

Advances in

Operations Research

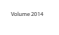

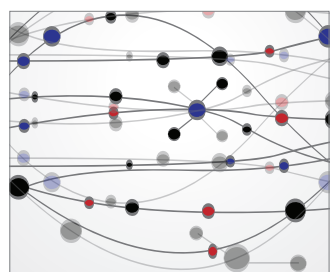

\section{The Scientific} World Journal
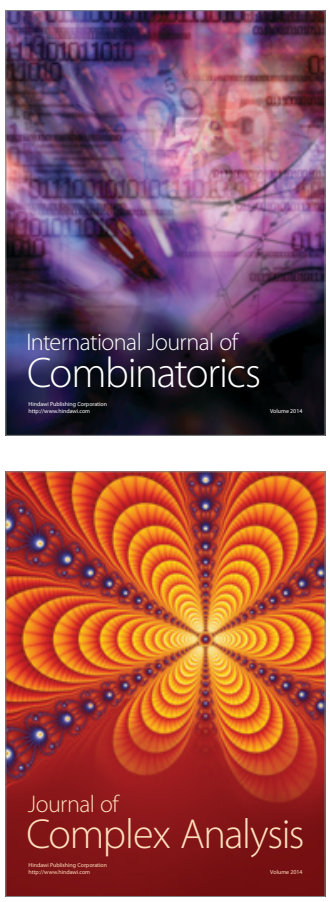

International Journal of

Mathematics and

Mathematical

Sciences
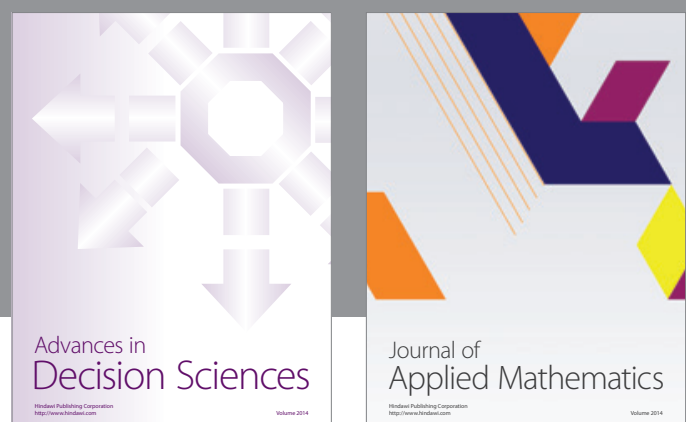

Journal of

Applied Mathematics
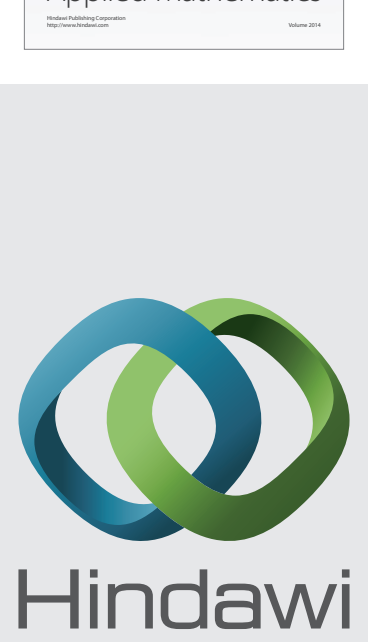

Submit your manuscripts at http://www.hindawi.com
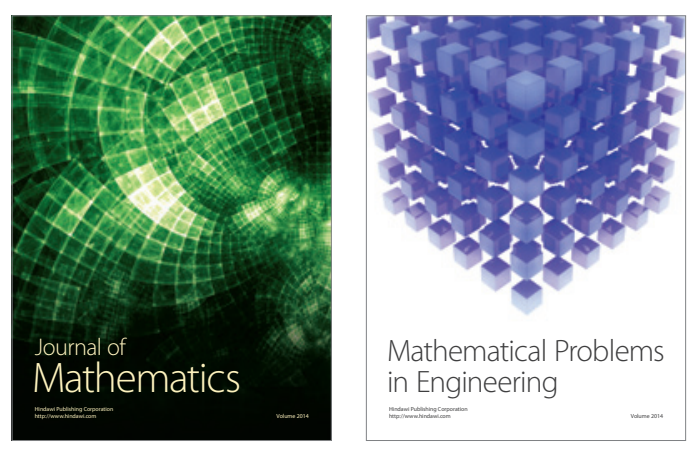

Mathematical Problems in Engineering
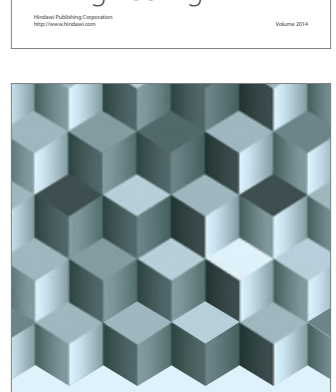

Journal of

Function Spaces
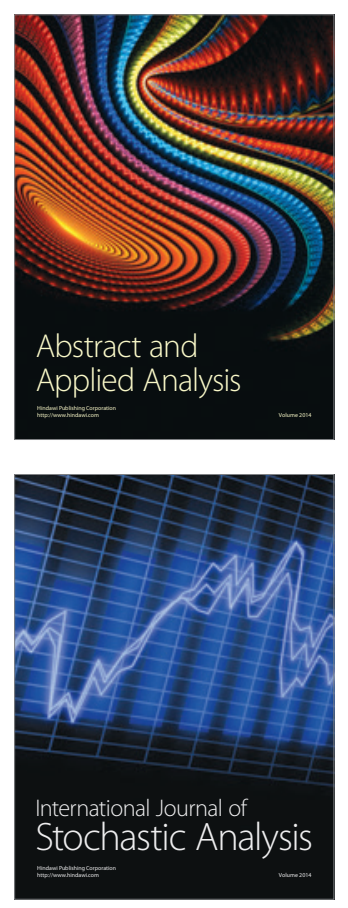

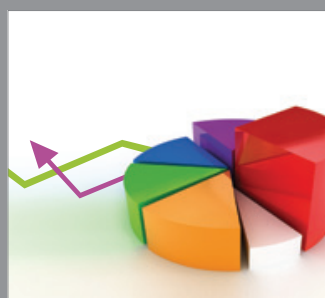

ournal of

Probability and Statistics

Promensencen
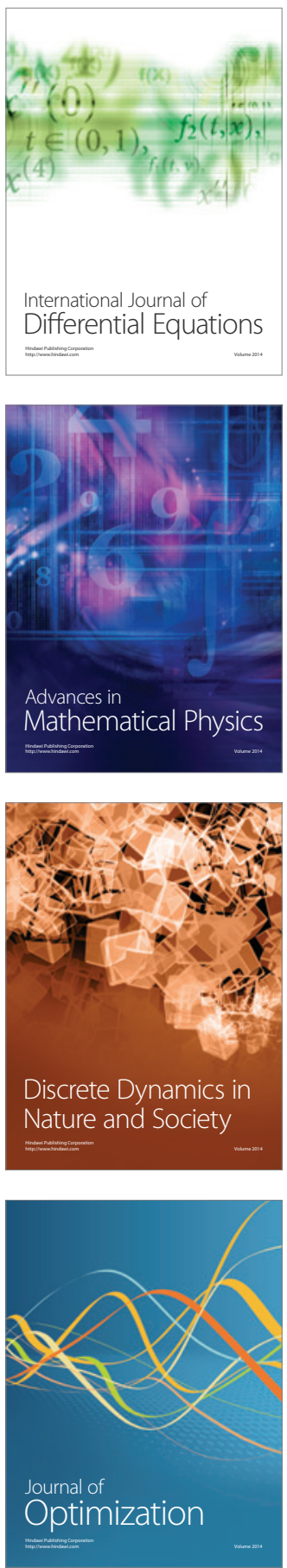